\title{
Application of Intelligent CAD in Landscape Design
}

\author{
Wei Zhang, Ming Tang, Juanjuan Yin \\ ZTE Telecommunication College, Xi'an Peihua Universty, Xi'an, 710125, China \\ 25100423@qq.com
}

Keywords: intelligent CAD; landscape design; expert system

\begin{abstract}
By analyzing the problems of the existing landscape design in engineering technology, expert system and intelligent CAD technology is applied to the architecture design of landscape, which satisfy the multiple tasks, multi-level and garden design requirements, and the expected function of the system is realized, the automation level of the process design is improved, it's also has a lot of help for the design of landscape system in actual application.
\end{abstract}

\section{Introduction}

Greening has become an important part of urban construction, and the landscape design and construction is an important part of urban greening. Landscape design is a technology which is study how to deal with the complex relationship among nature, architecture and human activity by using art and technology, so that to achieve the perfect harmonious, good ecological and picturesque scenery of the state. Art design can be performed and improved well by the designer's artistic accomplishment, but it will face many problems in terms of engineering technology: high demands for engineering and technical staff; Due to the different climate and topography and other reasons for different areas, the design of landscape system cannot be replicated in other places, so that the reusability of landscape design is low[1]; Existing CAD (Computer Aided Design, CAD) is a Computer Aided Design, it can only give a single graphics, so that client can hardly see the effect before molding design, which is not conducive to technical personnel to communicate with customers and cause deviation of the idea of designers and customers[2].

In the design system combined with experts and computer, machine intelligence can help or replace human expert to process data, information and knowledge, then make design decisions in a broader range and higher level, greatly improving the level of automation design, and thus greatly improve the efficiency and quality of design work, consequently adapt to the demands of rapid and changeable product development. To make a lot of picture of hills, lakes and ancient architectural structures, so that will directly generate preliminary design, then the customers can make changes on the generated program until being satisfied with them. Intelligent $\mathrm{CAD}$, intelligent engineering, intelligent design is put forward under this background [3].

\section{Expert System}

Expert system is an important branch of artificial intelligence fields, which is a computer application system to use of expert knowledge to solve the complex problems in the application fields. In the design of system, the design of landscape involves wide knowledge, it is difficult to achieve the high requirements for an ordinary designer. Such as, in the aspect of plants, we should fully consider the regional difference, the difference of the north and south climate and the receptivity of between different plants, then can avoid unnecessary loss. The content of utility is very huge, it is difficult to consider very detailed just only depend on several designers. So expert system will play a good role at this time, we only need to import as much as possible knowledge in database, the computer system will select the suitable plants automatically or pointed out that we do not consider the problem to us[4]. 
In the design of garden, Rule Engine was selected to use the reasoning machine [5]. The Rule Engine originated in rule-based expert system, the rule-based expert system is one of the branch of expert system, which mimics human reasoning and use tentative method for reasoning, and using the terms that humans can understand to explain and prove its reasoning. Using it can separate business logic of business decision makers and technical decisions of application developers in the application system and bind the business decisions in a central database or other place, they can manage and modify dynamically when they running., so as to provide effective technical support for maintain flexibility and competitiveness for the enterprise. Simply say: letting the business logic of the application can be treated by rule configuration.

\section{Intelligent CAD}

Traditional computer-aided design(CAD) technology cannot provide effective support for the intelligence activities of design engineer, and therefore it is no longer suited to the needs of modern product design and development. In order to improve the ability to respond quickly to market changes, small quantity and multi-variety requirements, design is developing to the directions of integration, intelligence and automation. To achieve this goal, we must greatly improve the level of intelligence of machine in the man-machine design system which is a combination of design experts and computer tools, so that the computer can help or in place of human experts to process data, information and knowledge in a wider range and a higher level, and make all kinds of design decisions, to greatly improve the level of design automation and efficiency and quality of design work, to adapt to the needs of market about rapidly changing of product development.

Intelligent CAD system is to strengthen the application of artificial intelligence techniques in all aspects of the design process, in particular to organically combine the expert system technology based on logical thinking with artificial neural network technology based on thinking in images to further improve the intelligence level of system [6]. Only after the field trip in terrain can the early garden design produce rendering that is a good match for this terrain by the AUTOCAD software. It causes that the difficult communication of technicians and customers and the production results have little reuse. The use of intelligent CAD can change the status. We can produce many graphics in system, such as rockery, lakes, ancient buildings and so on, and directly generate the preliminary design program in system, then allow customers to modify the generated program until get the satisfaction of customers.

\section{Architecture of Intelligence Garden System}

4.1 Architecture. For the features of "network, integration, intelligence and collaboration" of high-tech engineering development in the network environment, research and development for engineering design integrated development platform that groups of people of different roles participate in[7].

4.2 Data Base System (DBS). Establish the database according to the various data in landscape construction. The database should be included in the flowers, trees, landscape, pavilions, roads experts and so on[8].

The question library is a collection of problems, which is an index model. Actually, each question corresponds to a class of problems, such as garden style, layout scheme, pavilion, garden, landscape, recreation, scenery, the window etc. In the CAD data model, a specific data which is isolated often has been nonsense, but only a collection of related data can describe a complex thing. According to the trunk of information model which include relevant number, and supplemented by other information, these establish a complete and hierarchy-oriented structural diagram model. As shown in Figure 1. 


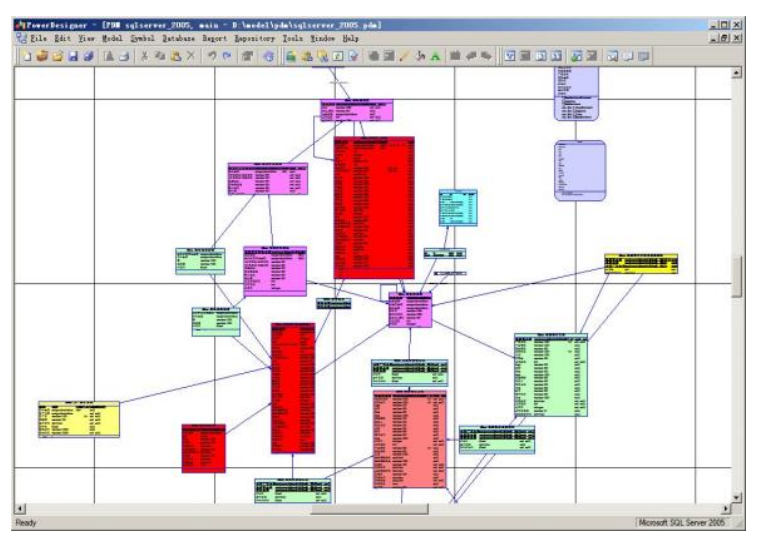

Figure 1. Database diagram

4.3 Model Library System. Model library is the foundation of 2D or 3D computer graphics modeling. It can establish an integral part of the design drawings. It includes many 2D/3D models in garden designing, such as terrain, trees, flowers, architecture.

4.4 Knowledge Library Systems. Firstly, it implements knowledge representation of engineering development working mode and overall control, which supports parallel and collaborative design environment. Secondly, it establishes global knowledge library that orient engineering process control by using the appropriate knowledge representation method. Finally, it designs a multi-agent reasoning mechanism for engineering collaborative designing, which implements automatic deduction of the design process by artificial intervention.

4.5 User Interface System. This system includes universal control programs and strongly interactive user interface. The visualization interactive environment of multidimensional information reflects in the character, graphics, audio, video interaction etc. It integrates with the co-user and multi-library system (knowledge library system, model library system, and database system), collaborative design process control components and collaborative design process management, and other components. We have researched the engineering management visualization and implemented automatic tracking of engineering design and development and engineering node content and progress visualization to supply decision information for engineering master. Decision maker and co-user may control multi-library system and other components by using visualization interface of multidimensional information, and manage collaborative design process on the macro[9].

4.6 Methods. (1)Based on the fundamental theory and method of the intelligent decision support system, closely integrated with engineering design, develop intelligent DSS for engineering field in practice, enrich the theory and method of decision support system, and broadening the research field of intelligent decision support system, lays the foundation for the automation development project under the environment of network[10].

(2) Establish the Object-oriented general integrated development platform. Different engineering has its special technical requirements, system will be more practical and efficient if we pay more attention to special requirement, will be more conducive to promoting if we pay more attention to general technology.

Therefore, to define them as much as possible, when they are in the popularization and application, we will use the software reusable technology criterion, only change the special part of the software system to make it completed through promotion. We are going to define the research content in the project demonstration stage, that is: to establish engineering oriented intelligent DSS general structure, the network topology of parallel environment is based on the generalization .Using the same reasoning model to develop intelligent database system and process control oriented knowledge base, the knowledge representation methods do in form is unified ;database and model base of development tools and general structure, but the data content and each has different according to different application object .In this project, the multi-dimensional information interaction system is special ,but common develop data interface.

(3) The group collaborative design mode of parallel environment. Establish the concurrent design environment which is based on the TCP/IP Internet environment, realize the group collaborative design mode of parallel environment. 


\section{Intelligent Design of the Garden}

Completely according to the landscape planning and design process, this system simulated the landscape planning and design of each link by computer. Every link can not only become isolated, but also dynamic associated to and influence the outcome of the other link. Convenient for each the designers of each link, the cooperation of the reviewers, the handover, and make the landscape design process can be modified by iteration.

According to the general landscape planning and design process, this software runs several subsystems as follows: Project profile set subsystem, needs to set the basic of the project, to determine the project automatically generated the basic rules; Terrain subsystem, its goal is to calculate with the inputs of geological survey data according to the inference machine, and output target garden landscape design; Main pathway system, its goal is to generate the trunk road with the output data of terrain subsystem; Construction subsystem, the goal is to generate the botanical garden in architectural design; Trees, flowers subsystem, the goal is to generate the pattern of trees and flowers in the garden; Hydropower network subsystem, the goal is to design water supply power supply system the related lamps, which are matched with houses, toilet, fountain, irrigation systems.

Drools.NET is adopted in development as a rule engine.Drools.NET is the.net version of the Drools, PETE algorithm based on Charles Forgy open source business rules engine. According to the inference rules and business needs, the landscape design made the subsystems, such a Terrain subsystem is shown in figure 2, Road subsystem is shown in figure 3, Building subsystem is shown in figure 4 and the final renderings as shown in figure 5 .

Terrain subsystem, for example, general rules of inference are as follows:

R1: information parameters inputted into the subsystem parameters as input parameters of the reasoning machine, such as the scale of construction, investment scale, service object, the geographical position, temperature, etc. According to the basic design specification of general landscape green space ratio, water ratio and space proportion, construction area, the terrain modification cost proportion in the overall cost can be calculated, etc.

$\mathrm{R} 2$ : Input the contour map and the result of R1, according to the landscape design template database to calculate the recommended terrain, road map, etc.

R3: Use the recommended terrain contour map and the original contour map to calculate the total into and out of earthwork quantity. With earthwork norm table, calculate whether the cost is in line with the investment.

R4: According to the template database to divide it into green space landscape design, construction, water, land area with the recommended topographic map.

R5: Artificial adjustment of terrain templates, design details, zoning, etc., take it into the rules of the R2, R3 and R4 to iterative, until the satisfactory results are obtained.

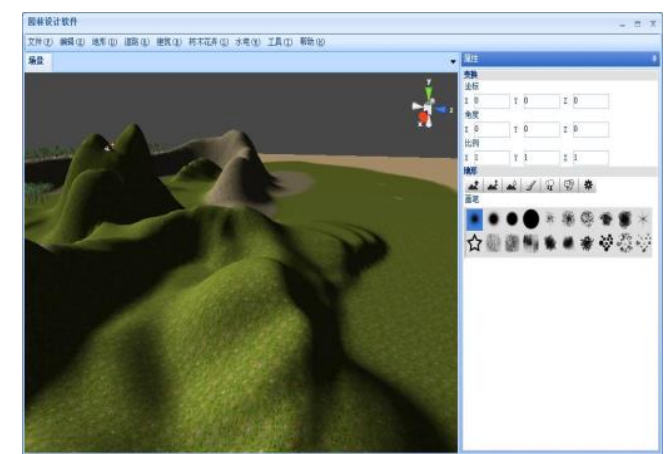

Figure 2. Terrain subsystem

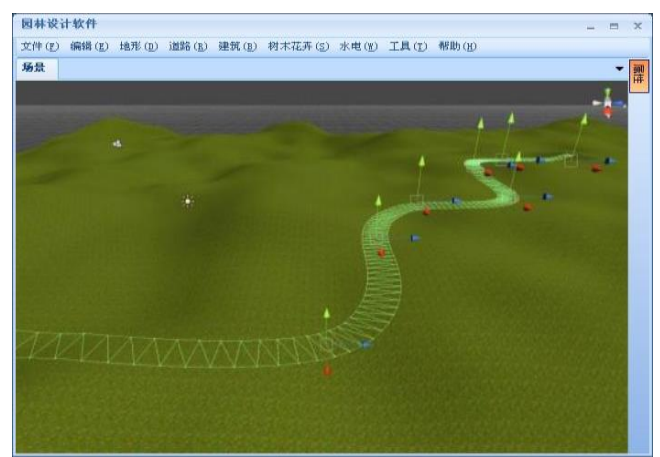

Figure 3. Road subsystem 


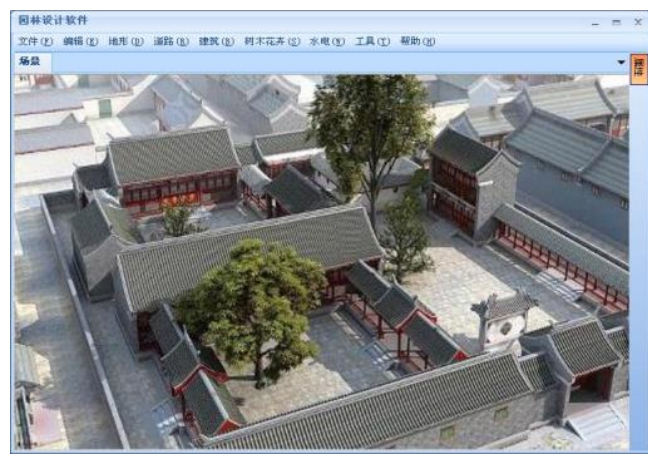

Figure 4. Building subsystem

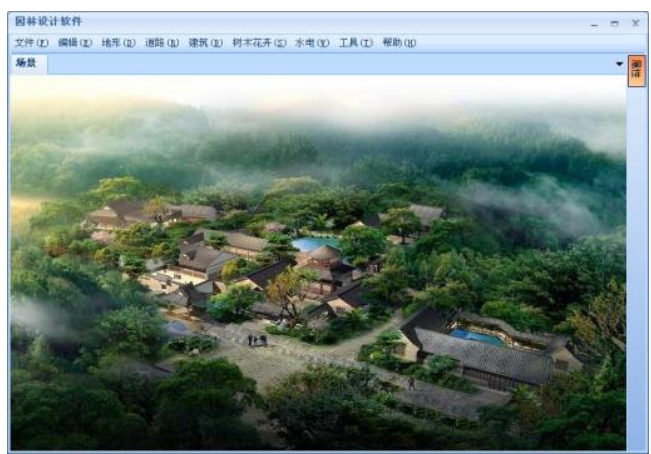

Figure 5. The final rendering

\section{Conclusion}

This paper introduced the intelligent CAD application in landscape design, from concept to design to specific implementation process. Intelligent CAD system not only requires CAD can provide complete landscape subsystem parameter information which includes the geographical position, such as temperature, light, monsoon winds but also requires to integrate these parameters and the system model together then establish a unified database. Intelligent CAD technology shortens the time to prepare for design, convenient for changing customer demand, At the same time design results can be directly shown, improved the landscape design of reuse and the competitiveness of the enterprises, But in landscape design, the need for various types of knowledge to seek appropriate expression should be taken into consideration, Especially for a large number of, discreteness, unstructured knowledge processing still has great limitations. If this kind of knowledge expressed with rules and framework, it will be a unusually large knowledge base, and make the program structure complicated. This is also the problem of intelligent CAD.

\section{References}

[1] Li Li, Lan zhipeng. Study on the Status of Mordern Landscape Architecture Planning [J]. Journal of Anhui Agricultural Science, 2009, 37(20):9763-9764.

[2] Pang yunling. Object-oriented Research and Development of Intelligent CAD System [D]. Tianjin Polytechnic University,2004.

[3] Zhang Zhaolin. The Development and Research Trends of Expert System and Intelligent CAD [J]. Forestry machinery and woodworking equipment, 2001, 08:4-7.

[4] Liu Bailin. Artificial Intelligence and Expert System[M]. Xi'an Jiaotong University Press, 2012.

[5] Ye Zhou, Wang Dong. Rules Engine Based Data Cleansing [J]. Computer Engineering, 2006, 32(23):52-54.

[6] Whang Jun. Key Techniques Research on Intelligent and Integrated CAD/CAPP System [D]. Yanshan University, 2010.

[7] Chen Lu, Tang Xueshan, Li Yong. The Ideal Landscaping Living Mode for Human Beings [J]. Journal of Beijing university of technology, 33(5): 555-560.

[8] Jiao Jingxin, Wang We, Tian bo. Database System Structure of Mixed Modes [J]. Journal of Detection and Control, 2014, 36(2):79-83.

[9] LI Xiao, QIU Yu-Hui. Evolution and Trends of Intelligent User Interfaces [J]. Computer Science, 2004,31(12): 122-125.

[10] Wei Qiang, Zhou Deyun. Research on UCAV's Intelligent Decision -Making System based on Expert System [J]. Fire Control and Command Control, 2007, 32(2):5-7. 